\title{
Magnetic and Thermodynamic Properties of the Three-Dimensional Periodic Anderson Hamiltonian
}

\author{
Carey Huscroft, ${ }^{1}$ A.K. McMahan, ${ }^{2}$ and R.T. Scalettar ${ }^{1}$ \\ ${ }^{1}$ Physics Department, University of California, Davis, CA 95616 \\ ${ }^{2}$ Lawrence Livermore National Laboratory, University of California, Livermore, California 94550
}

\begin{abstract}
The three dimensional periodic Anderson model is studied with Quantum Monte Carlo. We find that the cross-over to the Kondo singlet regime is remarkably sharp at low temperatures, and that the behavior of magnetic correlations is consistently reflected in both the thermodynamics and the density of states. The abruptness of the transition suggests that energy changes associated with the screening of local moments by conduction electrons might be sufficient to drive large volume changes in systems where applied pressure tunes the ratio of interband hybridization to correlation energy.
\end{abstract}

PACS numbers: 71.10.Fd, 71.10.Ht, 71.27.+a

The problem of localized, highly correlated electrons hybridizing with a conduction band is one of longstanding interest [1]. Our understanding of the underlying physics has recently been increased through new analytic approaches [1,2], and numeric methods like Quantum Monte Carlo (QMC) 3, 4 . These techniques have emphasized the connection between static magnetic properties and the dynamic response like the density of states.

However, what has been much less carefully explored by QMC is the link to thermodynamics. An intriguing problem for which a detailed understanding of the thermodynamics is essential is the "volume-collapse" transition in rare earth metals. This phenomenon occurs with the application of pressure to certain Lanthanides and gives rise to first order phase transitions with unusually large volume changes (14\% for Cerium and $9 \%$ for Praseodymium) [5.6]. Accompanying the change in volume is a change in the magnetism: On the expanded, highly correlated, side of the transition, the $f$ electrons have well defined moments, while on the contracted, less correlated, side these moments disappear or are expected to disappear. The low-volume $\alpha$ phase of Ce is paramagnetic, as are the early actinides which are considered to be analogs for the collapsed rare earth phases [6].

Even the qualitative origin of this phenomenon is still under debate. One suggestion is that the pressureinduced change in the ratio of the interaction strength to bandwidth gives rise to a Mott transition of the $4 f$ electrons accompanied by loss of magnetic order [7]. An alternate proposition is that the rapid change in the $4 f-$ valence electron coupling leads to a "Kondo volume collapse" [8]. In both cases, there are dramatic thermodynamic (e.g., pressure-volume) as well as magnetic signatures of the phenomenon.

In this paper we will establish the connection between the thermodynamics and the magnetic properties of the symmetric periodic Anderson model (PAM) in three dimensions. While previous efforts have focussed on the
Anderson impurity model [8.99, the capabilities of modern massively parallel computers now make feasible rigorous QMC calculations for the more realistic periodic model, which has so far received little attention in three dimensions. Our key results are:

- The dependence of the singlet correlation function on the interband hybridization shows an increasingly sharp structure as the temperature is lowered, indicating a very rapid cross-over between a regime where the $f$ sites have unscreened moments and one in which the moments are quenched by the conduction electrons.

- A sharp thermodynamic feature exists at the same interband hybridization as this change in the singlet correlator. To analyze this, we introduce a new approach to the calculation of the free energy $F$, and show it obeys various analytic sum-rules.

- The pressure difference at the transition inferred from $F$ is reasonably consistent with experimental pressure-volume data on $\mathrm{Ce}, \mathrm{Pr}$, and $\mathrm{Gd}$, given the approximate representation of the electronic structure.

The periodic Anderson Hamiltonian is

$$
\begin{aligned}
H= & \sum_{k \sigma} \epsilon_{k} d_{k \sigma}^{\dagger} d_{k \sigma}+\sum_{k \sigma} V_{k}\left(d_{k \sigma}^{\dagger} f_{k \sigma}+f_{k \sigma}^{\dagger} d_{k \sigma}\right) \\
& +U_{f} \sum_{i}\left(n_{i f \uparrow}-\frac{1}{2}\right)\left(n_{i f \downarrow}-\frac{1}{2}\right) \\
& +\sum_{i \sigma} \epsilon_{f} n_{i f \sigma}-\mu \sum_{i \sigma}\left(n_{i f \sigma}+n_{i d \sigma}\right) .
\end{aligned}
$$

We choose a simple cubic structure for which,

$$
\begin{aligned}
\epsilon_{k} & =-2 t_{d d}\left[\cos k_{x} a+\cos k_{y} a+\cos k_{z} a\right], \\
V_{k} & =-2 t_{f d}\left[\cos k_{x} a+\cos k_{y} a+\cos k_{z} a\right],
\end{aligned}
$$

where $a$ is the lattice constant. The dispersion of $V_{k}$ reflects our choice of near-neighbor (as opposed to onsite) hybridization of the $f$ and $d$ electrons [10]. Parameter values and temperature $T$ in this work are given in units of $t_{d d}$. We take $U_{f}=6$, consistent with the rare 
earths for a reasonable choice of $t_{d d}=1 \mathrm{eV}$, and explore a range of $t_{f d}$ and $T$ values. Our choice of the "symmetric" PAM dictates $\mu=\epsilon_{f}=0$, and thus half-filling: $\left\langle n_{i f}\right\rangle=\left\langle n_{i d}\right\rangle=1$. QMC results for this model were obtained using the determinant algorithm [11], which provides an exact treatment (to within statistical errors and finite size effects) of the correlations. There is no "sign problem" for the symmetric PAM, allowing accurate simulations at low temperatures.

Figure 1 shows the temperature and $t_{f d}$ dependence of the singlet correlation function of near-neighbor sites $\mathbf{i}, \mathbf{j}$,

$$
c_{f d}=\left\langle\vec{S}_{f \mathbf{i}} \cdot \vec{S}_{d \mathbf{j}}\right\rangle .
$$

Here $\vec{S}_{f \mathbf{i}}=\left(\begin{array}{ll}f_{\uparrow \mathbf{i}}^{\dagger} & f_{\downarrow \mathbf{i}}^{\dagger}\end{array}\right) \vec{\sigma}\left(\begin{array}{c}f_{\uparrow \mathbf{i}} \\ f_{\downarrow \mathbf{i}}\end{array}\right)$ and similarly for $\vec{S}_{d \mathbf{j}}$. For weak interband hybridization, $c_{f d}$ is small and the $f$ moments are unscreened by the conduction electrons. At low temperature, a sharp change is seen to occur at $t_{f d} \approx$ 0.6 to a phase where such screening is well established.

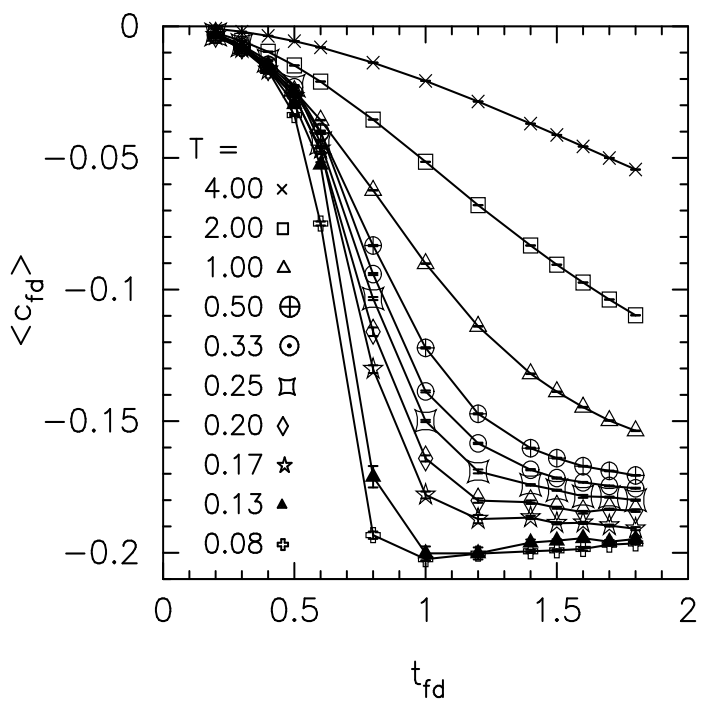

FIG. 1. The singlet correlation function $\left\langle\vec{S}_{f}(\mathbf{i}) \cdot \vec{S}_{d}(\mathbf{j})\right\rangle$ as a function of $f-d$ hybridization. As the temperature is lowered, there is an increasingly rapid switch from a small $t_{f d}$ regime where singlet correlations are absent to a large $t_{f d}$ regime where Kondo singlets are well formed.

This sharp switch is also reflected in the energy $E$ and free energy $F$. The difference $\Delta E(T)=E_{\mathrm{QMC}}(T)-$ $E_{\mathrm{AFHF}}(T)$ of the QMC calculations relative to antiferromagnetic Hartree-Fock (AFHF) results at the same temperature $(T=0.08)$ is shown in Fig. 2. To get $F=E-S T$ we fit [12] the raw data for $E_{\mathrm{QMC}}(T)$,

$$
E_{\mathrm{QMC}}(T)=E_{0}+\sum_{n} c_{n} e^{-n \Delta / T} .
$$

The number of fitting parameters $\left(E_{0}, c_{n}, \Delta\right)$ was taken to be about half of the number of data points. The entropy is then [13],

$$
S(T)=S_{0}+\frac{1}{T} \sum_{n} c_{n}\left(1+\frac{T}{n \Delta}\right) e^{-n \Delta / T} .
$$

Fig. 3 shows a plot of the resulting free energy difference $\Delta F(T)=F_{\mathrm{QMC}}(T)-F_{\mathrm{AFHF}}(T)$. Independent fits $\left(E_{0}\right.$, $\left.c_{n}, \Delta\right)$ were performed for each $t_{f d}$, so that the smoothness of the resultant curves in Fig. 3 is one measure of the success of this procedure. Another is that our fit yields $\sum_{n} c_{n} / n \Delta$ to within $\sim 3 \%$ of the expected value [13] for $t_{f d} \geq 0.8$. This sum is smaller by $\ln 2$ to within $\sim 3 \%$ for $t_{f d} \leq 0.5$, reflecting magnetic disorder of the spins below our lowest temperature $(T=0.08)$ in this regime, and consequent validity of the fit only for $T \geq 0.08$.

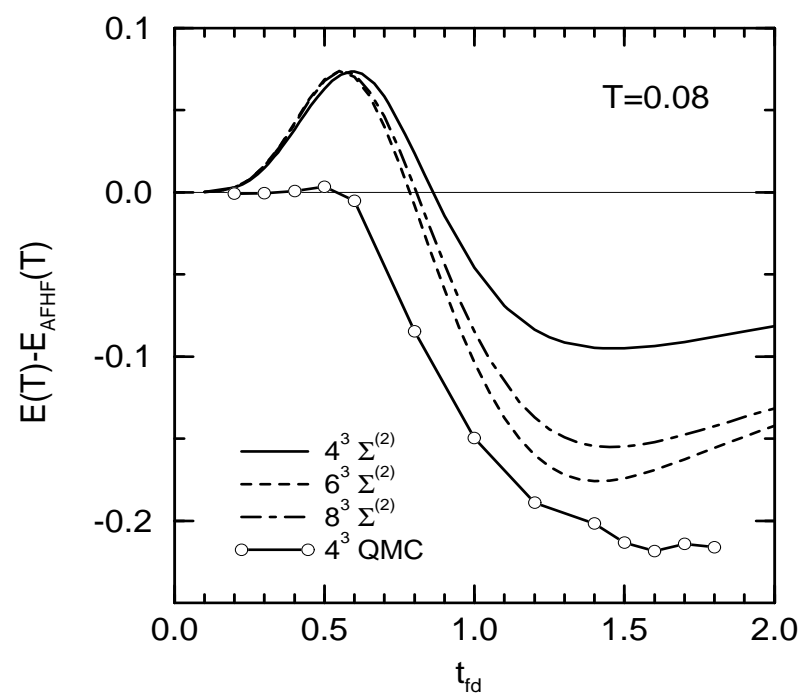

FIG. 2. The difference in energies between QMC and antiferromagnetic Hartree-Fock solutions. At small $t_{f d}$, the AFHF energy accurately tracks the QMC. However, at intermediate coupling the QMC results break away, reflecting the failure of $\mathrm{HF}$ to pick up the singlet correlations. A perturbation approach (labelled $\Sigma^{(2)}$ ) described in the text has some of the correct features seen in QMC.

The crucial feature in Figs. 2 and 3 is the rapid change in slope at low temperatures of $\Delta E$ and $\Delta F$ near $t_{f d}=$ 0.6. This behavior is hard to discern in the full thermodynamic functions whose variation with $t_{f d}$ is $\sim 20$ times larger than seen for these difference functions. It arises from the QMC results, and not from HF transitions, since the AFHF solution is stable throughout the $t_{f d}, T$ region plotted here. The size of the present slope change is not inconsistent with the volume collapse transitions, where one might view $\Delta F=\min \left(F_{1}, F_{2}\right)-F_{1}$ with $F_{1}$ and $F_{2}$ being free energy branches associated with the small $t_{f d}$ (large volume) and large $t_{f d}$ (small volume) phases, respectively. Given a volume dependence [6 of $t_{f d} \sim V^{-2}$, the slope change is related to a pressure difference by $V \Delta P / 2=-(1 / 2) \partial \Delta F / \partial \ln V \sim \partial \Delta F / \partial \ln t_{f d}$. Extrapolations of experimental pressure-volume data 14] into the two phase regions suggests $V \Delta P / 2 \sim 0.4,0.5$, and 
$1.3 \mathrm{eV}$ for $\mathrm{Ce}, \mathrm{Pr}$, and Gd, respectively. The low- $T$ slope change in Figs. 2 and 3 is $\partial \Delta F / \partial \ln t_{f d}=0.2-0.3 \mathrm{eV}$, which given the crudeness of the present representation of the rare earth valence electrons is reasonably consistent.

The QMC calculations were carried out for a $4^{3}$ site lattice. As a systematic exploration of system size for these three-dimensional calculations would be prohibitive, we have used a second-order self-energy approach to estimate the size effects, as well as to explore what analytic approximations might be more suitable than HF to capture the thermodynamics. The solid $\left(4^{3}\right)$, dash-dot $\left(6^{3}\right)$, and dash $\left(8^{3}\right)$ curves in Fig. 2 labelled $\Sigma^{(2)}$ were obtained from a finite-T version of the selfenergy approach of Steiner et. al. [15]. The Dyson equation for the interacting Green's function matrix $G_{\mathbf{k}}$ is solved based on a second order (in $U_{f}$ ) expression for the self-energy $\Sigma_{\mathbf{k}}^{(2)}\left(\left\{G_{\mathbf{k}}^{(0)}\right\}\right)$, determined from the paramagnetic HF result $G_{\mathrm{k}}^{(0)}$. The trend in the $\Delta E_{\Sigma}^{(2)}$ curves for periodic clusters of $4^{3}, 6^{3}$, and $8^{3}$ sites suggests that finite size effects 16] do not alter the qualitative physics and, indeed, move the position of the transition in the correct direction for comparison with experiment, namely to higher values of $\partial \Delta F / \partial \ln t_{f d}$.

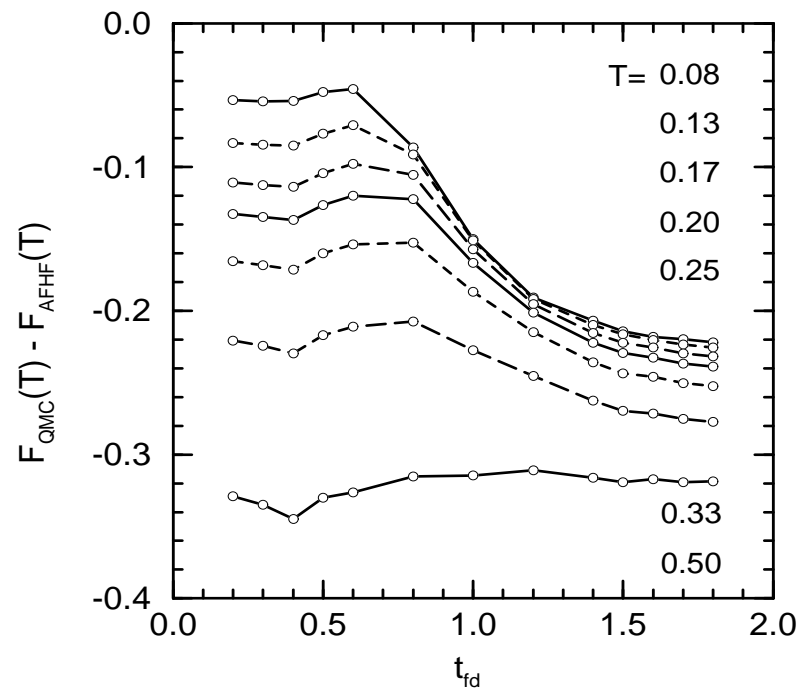

FIG. 3. The difference in free energies between QMC and antiferromagnetic Hartree-Fock solutions. At strong coupling $\left(\right.$ small $\left.t_{f d}\right)$ the agreement in the free energy is good apart from an overall shift of $T \ln 2$ associated with the tendency of HF to overestimate the magnetic order. As in Fig. 2, at intermediate coupling $\Delta F$ becomes sizeable.

There is striking consistency between the singlet correlations in Fig. 1 and the energy and free energy differences in Figs. 2,3. In all cases there is a rather abrupt switch in low-T behavior across $t_{f d} \sim 0.6$, which anneals with increasing temperature. The anomalies are largely gone above $T \sim 0.5$, an upper bound for what might be a critical temperature in the present model. The actual critical temperature will reflect competition between effects like these in $\Delta F$ and the volume dependence of a realistic generalization of $F_{\mathrm{AFHF}}$. An important term in $\Delta F$ is the QMC entropy, which reflects disordered spins for small $t_{f d}$ at the lowest temperature $T=0.08$, in contrast to both larger $t_{f d}$ values at this temperature, as well as the stable AFHF solution throughout the range plotted in Fig. 3, where the entropy is approximately minimal. Consequently, $\Delta F$ includes a $-T \ln 2$ entropy term at small $t_{f d}$, but not at large $t_{f d}$, which serves to level out the $\Delta F$ curves as temperature is increased.

Besides singlet formation, magnetic ordering of the local moments is a generic feature of the PAM. Indeed, our calculations of the $f-f$ structure factor suggest a strong tendency for the $f$ moments to order antiferromagnetically at low temperatures with a maximal ordering temperature in the vicinity of $t_{f d}=0.8$. Further insight into the relation between AF, singlet formation, and the thermodynamics can be obtained by computing the heat capacity $C(T)=d E(T) / d T[6]$. We find a low-temperature peak similar to recent work on the two-dimensional Hubbard model [12], with an area $\int d T C(T) / T$ of $\ln 2$ at small $t_{f d}$, which, however, washes out with decreasing area at large $t_{f d}$. The peak has only minor impact on the slope change discussed above for $\Delta E_{\mathrm{QMC}}$ and $\Delta F_{\mathrm{QMC}}$ as functions of $t_{f d}$ [17].

A more complete picture of the PAM is given by the density of states, $N_{f}(\omega)$, which we obtain using the Maximum Entropy method [18] to perform the analytic continuation of the imaginary time Greens function computed in QMC. The results for different $t_{f d}$ at fixed $T=0.2$ are shown in Fig. 4. $N_{f}(\omega)$ evolves from a structure with upper and lower Hubbard bands separated by a gap $U_{f}$ at small $t_{f d}$ to a regime where broadened remnants of these bands are still evident but additional resonant peaks characteristic of Kondo singlet formation have also developed. As $t_{f d}$ is increased, central resonances appear and are sharpest at $t_{f d} \approx 0.6$, indicating the onset of singlet formation. Further increase in $t_{f d}$ enhances the weight in this central region at the expense of the Hubbard sidebands.

The precise nature of the gap in the density of states at the Fermi surface, $\omega=0$, is still open to interpretation. For the half-filled, single band Hubbard Hamiltonian, $N(\omega)$ has a similar gap which evolves continuously from predominantly Mott-Hubbard character, for $U>>W$, to a Slater gap associated with antiferromagnetic order, for $U<<W$. Similarly, the two-band model considered here has a Mott gap at small $t_{f d}$, while the gap at larger $t_{f d}$ could originate either as a result of long range antiferromagnetic order on the $f$ sites, or, alternately, reflect a "coherence gap" associated with singlet formation. The competition between these two latter effects on $N(\omega)$ is well documented in a lower dimension [4]. Here, studies of the $f-f$ correlation function show no signs of $\mathrm{AF}$ 
long-range order at $t_{f d}=0.6$ and $T=0.2>T_{\text {Neel }}$, which suggests these resonances signal singlet formation, not AFLRO. Analytic continuation of two particle Green's functions, like the magnetic susceptibility, will lend further insight into this question, but is very difficult and remains to be done.

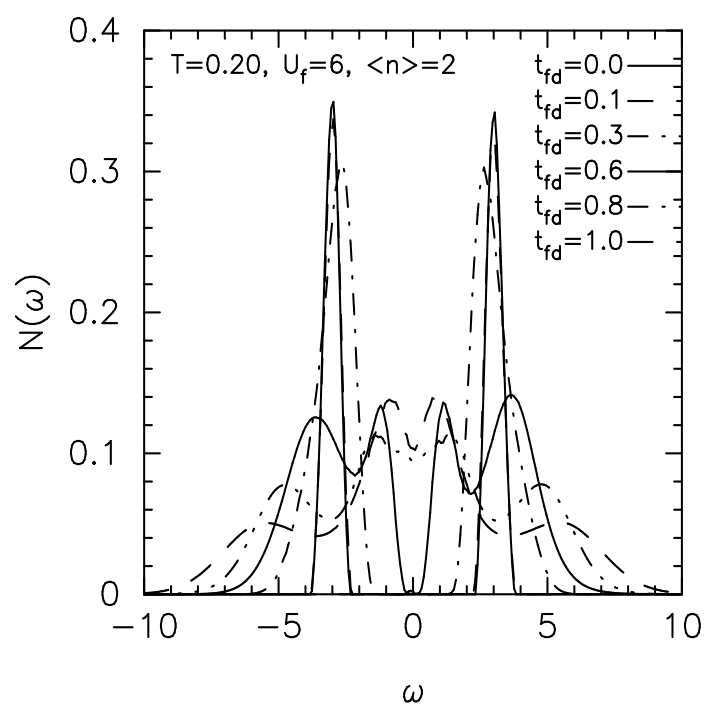

FIG. 4. The $f$-band density of states for different $f-d$ hybridization. For weak hybridization, there are peaks at $\pm U_{f} / 2$. These broaden with increasing $t_{f d}$, and a Kondo resonance develops. The curves for the two smallest $t_{f d}$ values have been reduced by a factor of two for display purposes.

In this paper we have shown that there is a striking consistency between the location of sharp cross-overs in the singlet magnetic and thermodynamic properties of the three-dimensional periodic Anderson model. The $f$ density of states shows a structure expected to arise from singlet correlations. Finally, estimates of the associated change in free energy are of the same order of magnitude as observed in the rare earth volume collapse transitions.

Two important issues remain open. The first is the extension to Hamiltonians with the full rare earth orbital complexity. Initial studies of how the Mott transition varies with band degeneracy in the Hubbard model, and other issues, already exist 19] within approximate numerical approaches like dynamical mean field theory [2]. The second, related, issue concerns band filling. Studies with many $f$ orbitals will require working away from the symmetric point.

Work at UCD was supported in part by an Accelerated Strategic Computing Initiative grant and by the LLNL Materials Research Institute; that at LLNL, by the U.S. Department of Energy under Contract No. W7405-Eng-48. The QMC calculations were performed on the ASCI Blue-Pacific and Red platforms.
[1] G.R. Stewart, Rev. Mod. Phys. 56, 755 (1984); P.A. Lee et.al., Comm. Cond. Matt. Phys. 12, 99 (1986); A.C. Hewson, "The Kondo Problem to Heavy Fermions," Cambridge Univ. Press, Cambridge (1993).

[2] D. Vollhardt, in Correlated Electron Systems, V. J. Emery ed. (World Scientific, Singapore) 57 (1993); A. Georges et.al., Rev. Mod. Phys. 6813 (1996); Th. Pruschke et.al., Adv. Phys. 44, 187 (1995); H. Tsunetsugu et.al. Rev. Mod. Phys. 69, 809 (1997).

[3] J. Bonča and J.E. Gubernatis, unpublished (condmat/9806374); Y. Zhang and J. Callaway, Phys. Rev. B 38, 641 (1988); M. Jarrell, Phys. Rev. B 51, 7429 (1995); A.N. Tahvildar-Zadeh et.al., Phys. Rev. B 55, 3332 (1997); M. Rozenberg, Phys. Rev. B52, 7369 (1995).

[4] M. Vekic et.al., Phys. Rev. Lett. 74, 2367 (1995).

[5] See, e.g., U. Benedict et.al., Physica 144B, 14 (1986).

[6] A. McMahan et.al., unpublished (cond-mat 9805064).

[7] B. Johansson, Philos. Mag. 30, 469 (1974).

[8] J.W. Allen and R.M. Martin, Phys. Rev. Lett. 49 1106, (1982); J.W. Allen and L.Z. Liu, Phys. Rev. B 46 5047, (1992); M. Lavagna et.al., Phys. Lett. 90A, 210 (1982); J. Phys. F 13, 1007 (1983).

[9] O. Gunnarsson and K. Schonhammer, Phys. Rev. B28 4315 (1983).

[10] With a momentum independent $V_{k}$, the PAM is a band insulator at half-filling at $U_{f}=0$. Our choice has no such hybridization gap and is therefore more representative of the rare earth metals.

[11] R. Blankenbecler et.al, Phys. Rev. D24, 2278 (1981).

[12] For a method which fits separate polynomials at low and high temperatures for the 2-d single band Hubbard model, see D. Duffy and A. Moreo, Phys. Rev. B55, 12918 (1997).

[13] The constant $S_{0} \equiv 4 \ln 2-\sum_{n} c_{n} / n \Delta$, and should equal $\left(2 N_{0} / N\right) \ln 2$ where $N_{0}$ is the number of discrete $k$ points which have $\epsilon_{k}=V_{k}=0$ on a finite lattice. $N_{0} / N \rightarrow 0$ as $N \rightarrow \infty$.

[14] See Fig. 1 and references in Ref. [6].

[15] M.M. Steiner et.al., Phys. Rev. B43, 1637 (1991).

[16] Local quantities like the energy and singlet correlations show relatively smaller finite size effects.

[17] One way to see this is from Fig. 2. The paramagnetic $\Sigma^{(2)}$ calculation in Fig. 2 has no low- $T$ peak in $C(T)$ yet yields structure similar to the full QMC results. Moreover, with guidance from the temperature dependence of $E_{\Sigma^{(2)}}(T)$, one may extrapolate low- $T$ "QMC" energies for which the $C(T)$ peak has been artificially removed. These modified QMC energies yield a $\Delta E_{\mathrm{QMC}}$ curve only slightly different from that in Fig. 2.

[18] M. Jarrell and J.E. Gubernatis, Phys. Rep. 269135 (1996).

[19] M.H. Hettler et.al, unpublished cond-mat/9803295); H. Kajueter and G. Kotliar Int. J. Mod. Phys. B11, 729 (1997); O. Gunnarsson et.al., Phys. Rev. B56 1146 (1997); J. Bunemann et.al., Phys. Rev. B57, 6896 (1998); Y. Motome and M. Imada, J. Phys. Soc. Japan 661872 (1997). 